\title{
Acute liver failure due to previously unrecognised dilated cardiomyopathy in a 17-year-old boy with Duchenne muscular dystrophy
}

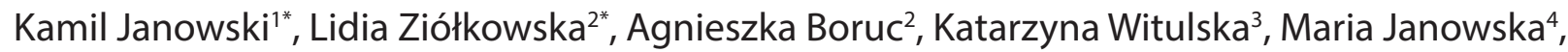 \\ Marek Stefanowicz ${ }^{4}$, Wioletta Jarmużek ${ }^{5}$, Joanna Pawłowska ${ }^{1}$ \\ 'Department of Gastroenterology, Hepatology, Nutritional Disorders, and Paediatrics, The Children's Memorial Health \\ Institute, Warsaw, Poland \\ 2Department of Cardiology, The Children's Memorial Health Institute, Warsaw, Poland \\ ${ }^{3}$ Department of Anaesthesiology, The Children's Memorial Health Institute, Warsaw, Poland \\ ${ }^{4}$ Department of Paediatric Surgery and Organ Transplantation, The Children's Memorial Health Institute, Warsaw, Poland \\ ${ }^{5}$ Department of Nephrology, The Children's Memorial Health Institute, Warsaw, Poland \\ *These authors contributed equally to this work.
}

\section{ABSTRACT}

We present a case of fatal liver failure secondary to previously unrecognised dilated cardiomyopathy in a 17-year-old boy with Duchenne muscular dystrophy (DMD). Acute liver failure in the clinical course of decompensated dilated cardiomyopathy in patients with DMD is rare and carries a high mortality rate. Severe congestive heart failure should always be considered as a possible cause of acute liver failure. In our article we summarise current recommendations including diagnostic and therapeutic standards regarding cardiac care in patients with DMD. Regular cardiac evaluation according to recommendations may detect the early stage of dilated cardiomyopathy and allow the implementation of preventive pharmacological treatment. Multispecialist management increases the survival and provides a better quality of life for children with DMD.

KEY WORDS:

dilated cardiomyopathy, muscular dystrophy, congestive heart failure, liver failure.

\section{CASE REPORT}

A boy, aged 17 years and 6 months, with Duchenne muscular dystrophy (DMD) and intellectual disability, was admitted to the Department of Gastroenterology, Hepatology, Nutritional Disorders, and Paediatrics of The Children's Memorial Health Institute in Warsaw, Poland (CMHI), referred from the district hospital with the initial suspicion of liver failure secondary to hepatitis due to cholelithiasis.

His past medical history was as follows: a boy delivered naturally from full-term multiple pregnancy (the first of twins, birth weight $2850 \mathrm{~g}$, length $52 \mathrm{~cm}$ ). Diagnosed with DMD at the age of seven years based on the neurological symptoms, electromyography, and laboratory and genetic tests. Post-diagnosis the multidisciplinary care was recommended, i.e. steroid therapy with prednisone $10 \mathrm{mg}$ every two days, speech therapy, rehabilitation, and neurological and psychological care. In the family history, DMD was confirmed in boys on the mother's side and unknown muscle pathology had been recognised in the sister of the boy's father. Other autoimmune and liver diseases were excluded. In the period from 7-17 years

\section{ADDRESS FOR CORRESPONDENCE:}

Kamil Janowski, Department of Gastroenterology, Hepatology, Nutritional Disorders, and Paediatrics,

The Children's Memorial Health Institute, 20 Dzieci Polskich Ave, 04-730 Warsaw, Poland,

ORCID: 0000-0001-5138-9341; e-mail: k.janowski@ipczd.pl 
of age the patient did not attend regular follow-up visits to specialist clinics. He was also not under cardiological care.

\section{FIRST SYMPTOMS AND TREATMENT IN THE DISTRICT HOSPITAL}

Abdominal pain and postprandial vomiting appeared 30 days before hospitalisation. Due to suspicion of gastroesophageal reflux disease, proton pump inhibitors and itopride therapy were applied by a paediatrician, but without good effect. After a further three weeks, constant fatigue and deterioration of exercise tolerance were noticed. Other symptoms were observed four days before admission to the paediatrics unit (purpura, loss of appetite, reluctance to drink, decreased diuresis, and jaundice). No fever was observed. The patient was admitted to the hospital in severe general condition, with disturbance of consciousness, dehydration, palpable pain under right rib cost, oedema in the legs, jaundice and purpura on the torso and lower limbs, and contractures of the knee, elbow, and ankle joints. Blood pressure was low (90-107/60-73 mm Hg), sinus tachycardia was 100/min, and blood saturation was $92-96 \%$. The taking of psychoactive substances, drugs (including acetaminophen), and mushrooms were excluded.

In laboratory tests (Table 1) the following was found: indeterminate International Normalised Ratio (INR) and Activated Partial Thromboplastin Time (APTT), hypofibrinogenaemia, significantly increased aminotransferase

TABLE 1. Summary of laboratory data

\begin{tabular}{|c|c|c|c|c|c|c|c|c|}
\hline \multirow{2}{*}{ Parameter } & \multicolumn{2}{|c|}{ Day 0} & \multirow[t]{2}{*}{ Day 1} & \multirow[t]{2}{*}{ Day 2} & \multirow[t]{2}{*}{ Day 3} & \multirow[t]{2}{*}{ Day 6} & \multirow[t]{2}{*}{ Day 8} & \multirow[t]{2}{*}{ Day 11} \\
\hline & $a^{a}$ & $I^{\mathbf{b}}$ & & & & & & \\
\hline INR & $\infty$ & 3.65 & 3.25 & 2.16 & 1.76 & 1.42 & 1.41 & 1.3 \\
\hline PT (s) & $\infty$ & 36.25 & 32.55 & 22.43 & 18.56 & 15.21 & 15.15 & 14 \\
\hline APTT (s) & $\infty$ & 48.5 & 59.3 & 43.6 & 36.5 & 37 & 30.6 & 31.7 \\
\hline Fibrinogen ( $\mathrm{g} / \mathrm{l})$ & 0.2 & 0.9 & 0.4 & 0.93 & 1.0 & 1.74 & 0.98 & 2.75 \\
\hline D-dimers (ng/ml FEU) & 13,390 & 8398 & 7521 & 10542 & 7792 & 3699 & 2207 & 3686 \\
\hline Albumin $(\mathrm{g} / \mathrm{l})$ & 35.7 & 35.8 & 33 & 31 & 32.8 & 38.4 & 46.7 & \\
\hline Total protein (g/l) & 54 & 50.4 & 44.5 & 46.5 & 51.5 & 55.6 & & \\
\hline $\operatorname{ALT}(\mathrm{U} / \mathrm{I})$ & 753 & 527 & 400 & 295 & 231 & 160 & 128 & 160 \\
\hline AST (U/I) & 1066 & 830 & 570 & 355 & 232 & 118 & 137 & 313 \\
\hline GGTP (U/I) & 64 & 57 & 43 & 37 & 35 & & & \\
\hline Total bilirubin (mg/dl) & 15.1 & 12.6 & 11.6 & 12.2 & 12.6 & 13.6 & & 52.9 \\
\hline Direct bilirubin (mg/dl) & 7.3 & 9.59 & 9.1 & 9.9 & 9.8 & 10.7 & & 52.1 \\
\hline $\mathrm{LDH}(\mathrm{U} / \mathrm{I})$ & & 1186 & 838 & 647 & 514 & 496 & & \\
\hline Ammonia ( $\mu \mathrm{g} / \mathrm{dl})$ & & 88 & 39 & 49 & 73 & & & \\
\hline Glycemia (mg/dl) & 61 & 83.9 & 86 & 128 & 135 & 118 & & \\
\hline Urea $(\mathrm{mg} / \mathrm{dl})$ & 91 & 92 & 90 & 56 & 19 & 29 & 49 & 62 \\
\hline Creatinine (mg/dl) & 0.6 & 0.55 & 0.67 & 0.82 & 0.64 & 0.24 & 0.27 & 0.2 \\
\hline Uric acid (mg/dl) & & 18.4 & 14.4 & 12.4 & & & & \\
\hline $\mathrm{Na}(\mathrm{mmol} / \mathrm{l})$ & 117 & 117 & 118 & 136 & 136 & 134 & 137 & 135 \\
\hline $\mathrm{K}(\mathrm{mmol} / \mathrm{l})$ & 5.94 & 6.06 & 4.8 & 3.5 & 3.5 & 4.4 & 4.2 & 2.75 \\
\hline Serum pH & 7.39 & 7.44 & 7.35 & 7.43 & 7.4 & 7.32 & 7.36 & 7.24 \\
\hline WBC (G/I) & 13 & 15.4 & 13.6 & 10.6 & 13.8 & 12.6 & 15.8 & 29.9 \\
\hline $\mathrm{RBC}(\mathrm{T} / \mathrm{l})$ & 4.9 & 4.45 & 3.9 & 3.63 & 3.41 & 3.15 & 2.87 & 2.95 \\
\hline $\mathrm{Hb}(\mathrm{g} / \mathrm{dl})$ & 15.4 & 13.3 & 11.3 & 10.7 & 9.9 & 9.3 & 8.4 & 8.9 \\
\hline Hct (\%) & 41.6 & 36 & 32 & 31 & 29 & 28 & 26 & 25 \\
\hline $\mathrm{PLT}(\mathrm{G} / \mathrm{l})$ & 65 & 50 & 48 & 88 & 102 & 40 & 82 & 75 \\
\hline $\operatorname{CRP}(\mathrm{mg} / \mathrm{dl})(\mathrm{n}:<0.5)$ & $2.3(\mathrm{n}:<1)$ & 1.49 & 1.18 & 1.57 & 2.49 & 2.02 & 2.91 & 13.5 \\
\hline
\end{tabular}

${ }^{a}$ results in the local hospital, ${ }^{b}$ results after admission to CMHI, $\infty$ - extremely extended, out of the count, INR - international normalised ratio, PT - prothrombin time, APTT - activated partial thromboplastin time, ALT - alanine transaminase, AST - aspartate transaminase, GGTP - gamma-glutamyltransferase, LDH - lactate dehydrogenase, WBC - white blood cells, RBC - red blood cells, Hb - haemoglobin, Hct - haematocrit, PLT - platelets, CRP - C-reactive protein 


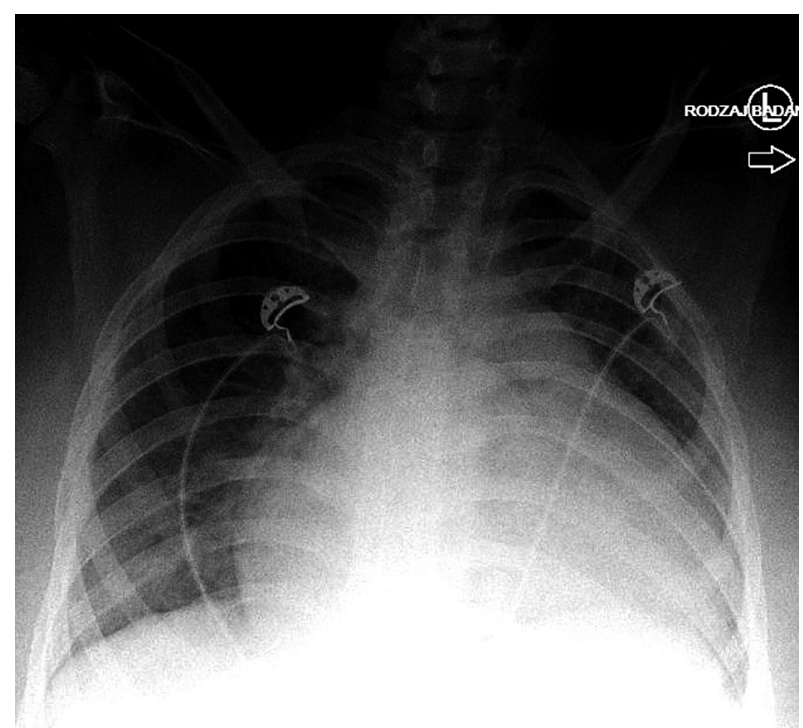

FIGURE 1. A chest radiograph with revealed enlarged heart

activity, hyperbilirubinaemia, normal albumin concentration, decreased total protein concentration, increased C-reactive protein (CRP), increased D-dimers, hypoglycaemia, leukocytosis and granulocytic smear, thrombocytopaenia, normal creatinine, elevated urea, hyponatraemia, and hyperkalaemia.

Despite intensive intravenous fluid therapy, administration of antibiotics (ceftriaxone), vitamin K supplementation, and fresh frozen plasma transfusions, no improvement was observed. Furthermore, in abdominal ultrasound examination, gallstones in the gall bladder and common bile duct dilatation (to $11 \mathrm{~mm}$ ) were described. Endoscopic retrograde cholangiopancreatography (ERCP) and cholecystectomy at the reference centre were recommended in the future. At the time, the patient was referred to $\mathrm{CMHI}$ with the initial suspicion of liver failure secondary to hepatitis due to cholelithiasis.

\section{TREATMENT AT THE REFERENCE CENTRE}

At the time of admission, the patient was in a severe general condition, with progressively decreasing consciousness (disorientation and confusion alternating with periods of agitations), with a motor response to touch, dehydration symptoms, jaundice, and massive oedema of the legs. At this time, the patient did not present symptoms of respiratory failure and he breathed unassisted.

In the neurology examination, slow pupil reaction to light and no deep reflexes were detected. Brain oedema, cerebral haemorrhage, and stroke were excluded based on CT of the brain, but, preventively, anti-oedema treatment was applied (20\% mannitol, furosemide).

In a repeated abdominal ultrasound examination, enlarged liver with high echogenicity, slightly heterogeneous, and gallbladder with thin wall and several gallstones (to $3 \mathrm{~mm}$ ) in the lumen was confirmed, with normal bile ducts and common bile duct widened to $4 \mathrm{~mm}$.
Normal renal and portal vein flow without ascites were observed.

Due to electrolyte imbalance, increased urea, uric acid levels, and oliguria $(0.3 \mathrm{ml} / \mathrm{kg} / \mathrm{h})$, the patient was consulted nephrologically. Intensive intravenous fluid therapy, correction of coagulation disorders, and treatment of hyponatraemia and hyperkalaemia were continued, while diuresis was forced and rasburicase (a recombinant urate-oxidase reducing levels of uric acid) was added to the therapy.

The results of laboratory tests are shown in Table 1.

As part of the differential diagnosis, we excluded bacterial infections by microbiological tests of blood, urine, and faeces. Infections of hepatotropic viruses, EBV, and HSV were excluded by serological tests. Because of weakly positive CMV IgM antibodies, the primary acyclovir therapy was changed to ganciclovir, but a PCR test did not confirm CMV viraemia.

Three hours after admission, further deterioration of the patient's general condition was observed, with increasing symptoms of encephalopathy. At this point, the patient was transferred to the Intensive Care Unit of the CMHI (ICU), where treatment and further diagnostics were continued.

Chest X-ray showed bilateral perihilar areas of increased opacity, a trace of fluid in the right pleural cavity, and a significantly enlarged heart with a cardio-thoracic ratio of 0.73 (Fig. 1).

In echocardiography typical features of dilated cardiomyopathy were found. The left ventricle was spherical and markedly enlarged (the surface area was $43.1 \mathrm{~cm}^{2}$ [norm 23.6-40 $\mathrm{cm}^{2}$ ]; $z$-score +2.5 ). Left ventricular diastolic diameter was $67 \mathrm{~mm}$ (norm 36.8-54.8 mm); $z$-score +4 ; and $148 \%$ of average value indexed to body surface area. Left ventricular contractility was extremely reduced (shortening fraction $10 \%$ [norm above $28 \%$ ], ejection fraction by Simpson 20\% [norm above 55\%]). The mitral valve regurgitation of third degree, significantly enlarged left atrium, and dilated pulmonary veins were found. The right ventricle was also enlarged (the surface area was $34.7 \mathrm{~cm}^{2}$ [norm 13.6-26.9 $\mathrm{cm}^{2}$ ]; $z$-score +3.2 ) with reduced contractility and fractional area change (FAC) value $23 \%$ (norm above $35 \%$ ). The tricuspid valve regurgitation of second degree was observed. The pericardial fluid behind the right atrium and right ventricle up to $15-18 \mathrm{~mm}$ and behind the left ventricle $3.5 \mathrm{~mm}$ was detected. Intensive heart failure treatment and intravenous positive inotropic drug (milirinone) was included with slightly improved left ventricle contractility (ejection fraction by Simpson 25-27\%) (Fig. 2).

In electrocardiography sinus tachycardia $100 / \mathrm{min}$, features of left ventricular volume overload and disturbances in the repolarisation period were found (Fig. 3).

Table 2 presents the results of biochemical markers of heart failure.

The results of cardiological tests (echocardiography, electrocardiography) and laboratory tests (Table 2) con- 
firmed severe heart failure. Multiple organ dysfunction syndrome was recognised because of liver, renal, and heart failure. The patient was intubated (mechanical ventilation was started) and he was qualified to CVVHDF (continuous veno-venous haemodiafiltration). In the next days, we observed increasing inflammation markers requiring antibiotic modification. Also, a dopamine and adrenalin infusion was necessary. The patient's general condition was deteriorating despite intensive management. Multiple organ failure and extremely severe general condition did not allow consideration of heart and/or liver transplantation and were a reason for disqualification from these procedures. On the $11^{\text {th }}$ day of ICU stay, in the absence of effective treatment and poor prognosis, the procedure for limiting futile therapy was initiated. The patient died 15 days after admission to the CMHI, due to cardiac arrest secondary to multiple organ dysfunction and circulatory failure caused by dilated cardiomyopathy associated with DMD. The autopsy was abandoned due to a known cause of death.

\section{DISCUSSION}

This article presents a case of a teenage boy with DMD and multiple organ failure secondary to dilated cardiomyopathy associated with DMD.

In our patient the diagnosis of dilated cardiomyopathy was made in a very late stage of the disease. It is worth

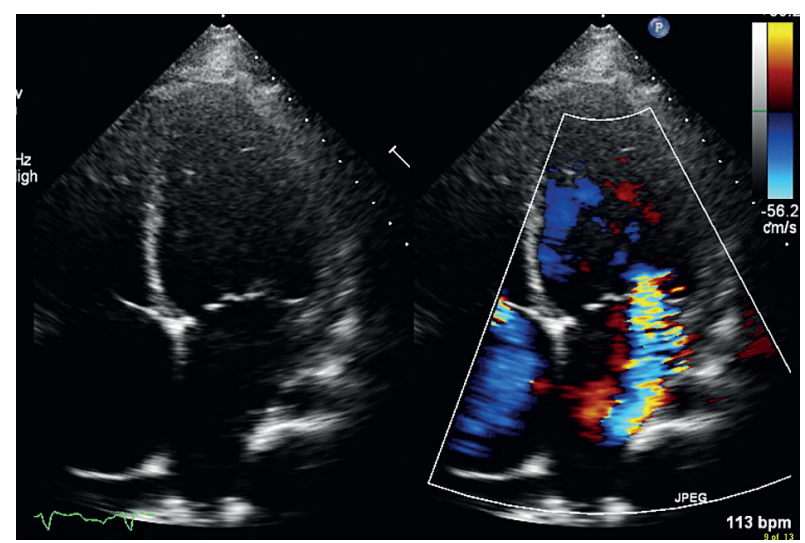

FIGURE 2. Echocardiographic examination, apical projection, fourchamber view. Left ventricular and left atrial enlargement, mitral valve regurgitation third

TABLE 2. The results of biochemical markers of heart and muscles failure

\begin{tabular}{|l|c|}
\hline Parameter & Value \\
\hline NTproBNP $(\mathrm{pg} / \mathrm{ml})(\mathrm{n}:<135)$ & 27,373 \\
\hline CK-MB $(\mathrm{ng} / \mathrm{ml})(\mathrm{n}:<6.6)$ & 180.2 \\
\hline $\mathrm{CTnl}(\mathrm{pg} / \mathrm{ml})(\mathrm{n}:<10)$ & $2 ., 5$ \\
\hline Myoglobin $(\mathrm{ng} / \mathrm{ml})(\mathrm{n}: 23-72)$ & 2730.2 \\
\hline CK (U/l) $(\mathrm{n}:<270)$ & 9745 \\
\hline
\end{tabular}

NTproBNP - N-terminal pro brain natriuretic peptide, $C K-M B$ - creatine kinase-MB subunit, cTnl - cardiac troponin I, CK - creatine kinase

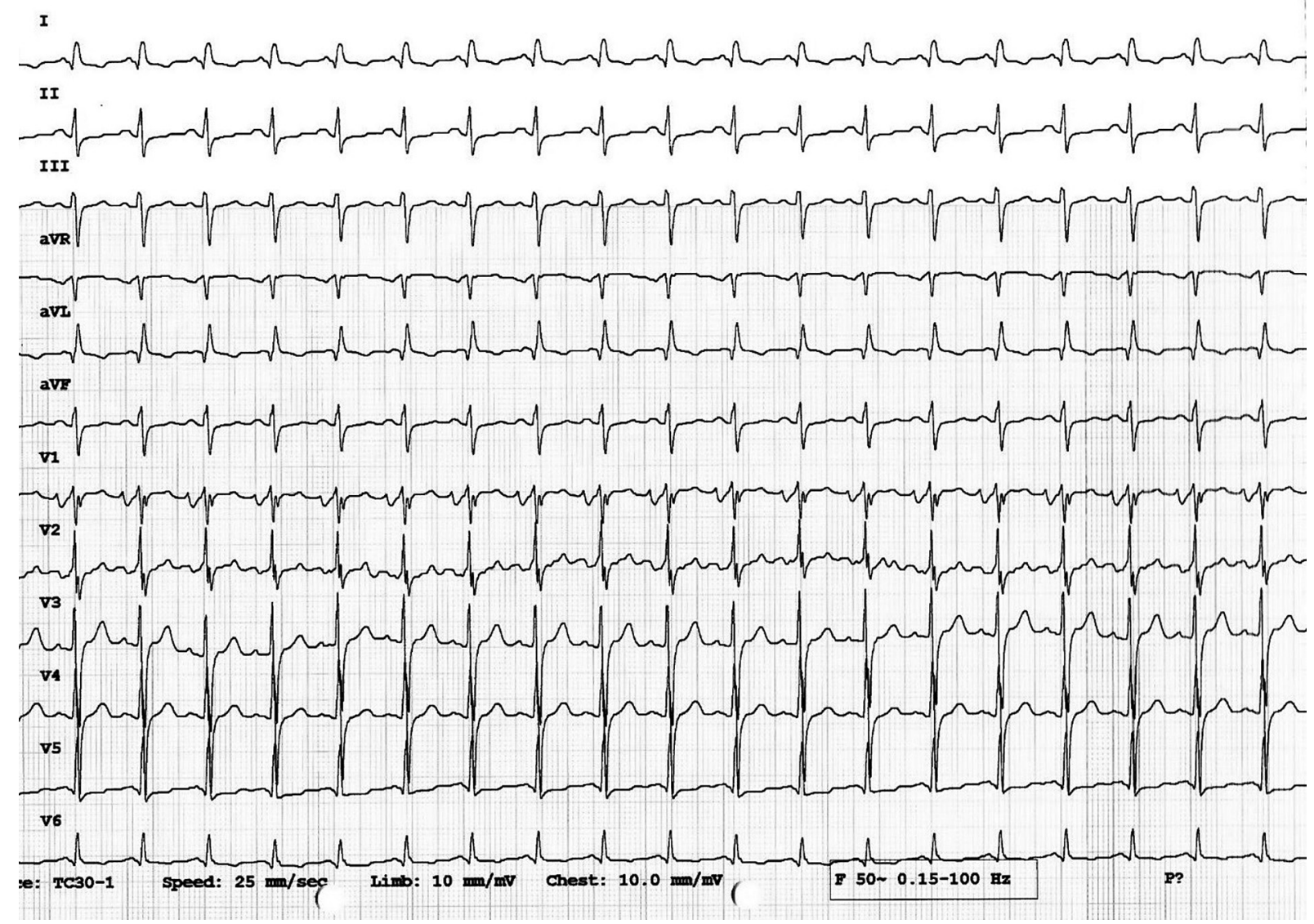

FIGURE 3. ECG with sinus tachycardia 100/min, features of left ventricular volume overload and disturbances in the repolarisation period 
noting that the patient had never been consulted by a cardiologist and had had no cardiac examinations, although the diagnosis of DMD was made at seven years of age. Another important fact in the management of our patient is that there were also other people diagnosed with DMD in the family. Hepatic failure was the primary reason for urgent hospitalisation, as the first symptom of multiple organ dysfunction. Renal failure and extreme decompensation of heart efficiency occurred shortly after that. Symptoms suggesting acute liver failure were the reason for referral of the patient to a reference centre treating children with acute liver failure. Based on laboratory tests and abdominal ultrasound results, in the district centre initially hepatic failure due to cholelithiasis was suspected. At that time, laboratory tests showed high alanine transaminase (ALT) and aspartate transaminase (AST) activity with hyperbilirubinaemia, disproportionate to relatively low gamma-glutamyltransferase (GGTP) activity, with initially undetectable coagulation parameters. In the comparative abdominal ultrasound, only single gallstones in the gallbladder and normal bile ducts were described. Therefore, a cholagogue cause of liver failure secondary to cholelithiasis was unlikely.

The result of echocardiographic examination showed features of dilated cardiomyopathy, with severe haemodynamic disorders in the form of left ventricular dilation and extremely impaired myocardial contractility. Despite intensive treatment for heart failure, the left ventricular systolic function was not improved and liver and kidney function could not be normalised.

In the medical literature, cases of acute liver failure secondary to multi-organ damage are well described [1-3]. The mechanism of liver failure based on congestive heart failure was presented in a few papers. Although the pathophysiology is poorly understood, there is rising evidence that low cardiac output with consecutive reduction in hepatic blood flow is a key causative factor, rather than hypotension. In the setting of acute liver failure due to congestive heart failure, clinical signs of the latter can be absent, which requires an appropriate diagnostic approach. In the literature issues, there are several reports of acute liver failure secondary to dilated cardiomyopathy in adults [4-7] and a few cases of previously unrecognised cardiomyopathy causing acute liver damage [8]. However, a case presenting acute liver failure due to previously unrecognised dilated cardiomyopathy in the course of DMD in adults, as well as in children, has not yet been published.

DMD is a severe neuromuscular disease of genetic origin that affects male children and is characterised by progressive muscle deterioration and accompanying cardiomyopathy. DMD is an X-linked genetic disorder in which mutations in the dystrophin gene destroy the link between the internal muscle filaments and the extracellular matrix, resulting in severe muscle weakness and progressive muscle wasting. That disorder affects about
13-33 per 100,000 males [9], and about $96 \%$ of DMD patients present symptoms of dilated cardiomyopathy [10]. Research results show that $25 \%$ of teenagers have symptomatic left ventricular systolic dysfunction [11].

Most patients with DMD develop cardiomyopathic features between the ages of 10 and 15 years. Dilated cardiomyopathy in patients with DMD is characterised by varying progression and is the leading cause of death among these patients. Because of this tight timeline during which heart dysfunction appears, DMD offers a unique opportunity to assess strategies to limit cardiomyopathy progression.

Medical management recommendations from the 2010 DMD Care Considerations document (updated in 2018) discuss the use of angiotensin-converting enzyme (ACE) inhibitors as first-line therapy once LV dysfunction has developed. However, initiation of therapy is often considered before ventricular dysfunction is detected, to delay the development of the disease onset. Angiotensin receptor blockers (ARBs) can readily be substituted for an ACE inhibitor in those who are intolerant of ACE inhibitors. In the last decade, a number of retrospective and prospective open, nonrandomised studies have suggested a benefit of $\beta$-blockade in DMD cardiomyopathy [12-18]. It was the opinion of experts that ACE inhibitor/ARB use in DMD should begin by 10 years of age, but the relatively low risk of ACE inhibitors and ARBs should not discourage the consideration of earlier prophylactic therapy. A recent study on mineralocorticoid receptor antagonism indicates that early use of these agents is helpful for DMD cardiomyopathy [19-21].

In 2018 The Lancet published recommendations providing a comprehensive set of multidisciplinary guidelines focused on the diagnosis and management of children diagnosed with DMD. Specific imaging recommendations included echocardiogram beginning at six years of age and then subsequent studies every one to two years, depending on age. After 10 years of age, it is recommended that DMD patients undergo annual echocardiograms. Cardiovascular magnetic resonance (CMR) has been more widely applied to DMD cardiomyopathy for its ability to characterise myocardial abnormalities. CMR with gadolinium is an additional emerging tool that is rapidly becoming the preferred method for cardiac monitoring in boys with DMD. Evidence for increased gadolinium enhancement on CMR is thought to reflect myocardial damage and fibrosis in the DMD heart [22]. Because myocardial fibrosis is one of the earliest findings of cardiac involvement in DMD, its presence has been used to support early institution of cardioprotective medications for DMD cardiomyopathy. It should be emphasised that experts recommend performing a baseline evaluation of patient with DMD at the time of diagnosis of the disease, which should absolutely contain consultation with a cardiologist, cardiac medical history, family history, physical examination, electrocardiogram, echocar- 
diogram $(<6-7$ years old), and cardiovascular magnetic resonance imaging ( $\geq 6-7$ years old) [23-25].

\section{CONCLUSIONS}

A multidisciplinary approach to care is essential for optimum management of the primary manifestations and secondary complications of DMD patients.

Contemporary, multispecialist management of children with DMD should include neurological, orthopaedic, rehabilitation, cardiological, gastroenterological, pulmonary, endocrine , and psychological care to increase their survival and improve their quality of life [26].

Regular cardiac evaluation according to recommendations, including routine monitoring of electrocardiograms, echocardiograms, and appropriate blood biomarkers, may detect early DCM development in patients with DMD.

The results of clinical trials and expert recommendations clearly provide evidence for early intervention to prevent or slow cardiomyopathy progression with medical management.

Acute liver failure induced by decompensated heart failure in patients with DMD is rare and carries a high mortality rate. Patients with acute liver failure require an appropriate multidisciplinary diagnostic approach. Severe congestive heart failure should always be considered as a possible cause of acute liver failure.

\section{DISCLOSURE}

The authors declare no conflict of interest.

\section{REFERENCES}

1. Correale M, Tarantino N, Petrucci R, et al. Liver disease and heart failure: Back and forth. Eur J Intern Med 2018; 48: 25-34.

2. Scarpati G, De Robertis E, Esposito C, Piazza O. Hepatic encephalopathy and cirrhotic cardiomyopathy in Intensive Care Unit. Minerva Anestesiol 2018; 84: 970-979.

3. Saner FH, Heuer M, Meyer M, et al. When the heart kills the liver: acute liver failure in congestive heart failure. Eur J Med Res 2009; 14: 541-546.

4. Fussell KM, Awad JA, Ware LB. Case of fulminant hepatic failure due to unrecognized peripartum cardiomyopathy. Crit Care Med 2005; 33: 891-893.

5. Wagle K, Akinseye OA, Shrestha P, et al. A Case of Fulminant Hepatic Failure Secondary to Congestive Heart Failure Without Evidence of Acute Cardiac Decompensation. S D Med 2017; 70 : 155-159.

6. Kaymakcalan H, Dourdourekas D, Szanto PB, Steigmann F. Congestive heart failure as cause of fulminant hepatic failure. Am J Med 1978; 65: 384-388.

7. Kisloff B, Schaffer G. Fulminant hepatic failure secondary to congestive heart failure. Am J Dig Dis 1976; 21: 895-900.

8. Wiesen S, Reddy KR, Jeffers LJ, Schiff ER. Fulminant hepatic failure secondary to previously unrecognized cardiomyopathy. Dig Dis 1995; 13: 199-204.

9. Karasek D, Sinkiewicz W. Problemy kardiologiczne chorych z dystrofiami nerwowo-mięśniowymi. Kardiol Dypl 2012; 11: 32-36.
10. Schade van Westrum SM, Hoogerwaard EM, Dekker L, et al. Cardiac abnormalities in a follow-up study on carriers of Duchenne and Becker muscular dystrophy. Neurology 2011; 77: 62-66.

11. Thrush PT, Allen HD, Viollet L, et al. Re-examination of the electrocardiogram in boys with Duchenne muscular dystrophy and correlation with its dilated cardiomyopathy. Am J Cardiol 2009; 103: 262-265.

12. Bourke JP, Watson G, Muntoni F, et al. Randomised placebo-controlled trial of combination ACE inhibitor and beta-blocker therapy to prevent cardiomyopathy in children with Duchenne muscular dystrophy? (DMD Heart Protection Study): a protocol study. BMJ Open 2018; 8: e022572.

13. Doing AH, Renlund DG, Smith RA. Becker muscular dystrophy-related cardiomyopathy: A favorable response to medical therapy. J Heart Lung Transplant 2002; 21: 496-498.

14. Bushby K, Muntoni F, Bourke JP. 107th ENMC international workshop: The management of cardiac involvement in muscular dystrophy and myotonic dystrophy. 7th-9th June 2002, Naarden, the Netherlands. Neuromuscul Disord 2003; 13: 166-172.

15. Rhodes J, Margossian R, Darras BT, et al. Safety and efficacy of carvedilol therapy for patients with dilated cardiomyopathy secondary to muscular dystrophy. Pediatr Cardiol 2008; 29: 343-351.

16. Viollet L, Thrush PT, Flanigan KM, et al. Effects of angiotensin converting enzyme inhibitors and/or beta blockers on the cardiomyopathy in Duchenne muscular dystrophy. Am J Cardiol 2012; 110: 98-102.

17. Kajimoto H, Ishigaki K, Okumura K, et al. Beta-blocker therapy for cardiac dysfunction in patients with muscular dystrophy. Circ J 2006; 70: 991-994.

18. Ogata H, Ishikawa Y, Ishikawa Y, Minami R. Beneficial effects of beta blockers and angiotensin-converting enzyme inhibitors in Duchenne muscular dystrophy. J Cardiol 2009; 53: 72-78.

19. Fairclough RJ, Bareja A, Davies KE. Progress in therapy for Duchenne muscular dystrophy. Exp Physiol 2011; 96: 1101-1113.

20. Sun G, Haginoya K, Dai H, et al. Intramuscular renin-angiotensin system is activated in human muscular dystrophy. J Neurol Sci 2009; 280: 40-48.

21. McNally EM, Kaltman JR, Benson DW, et al. Contemporary cardiac issues in Duchenne muscular dystrophy. Working Group of the National Heart, Lung, and Blood Institute in collaboration with Parent Project Muscular Dystrophy. Circulation 2015; 131: 1590-1598.

22. Kwiatkowska J, Meyer-Szary J, Bazgier M, et al. Left ventricular volumes and function affected by myocardial fibrosis in patients with Duchenne and Becker muscular dystrophies: a preliminary magnetic resonance study. Kardiol Pol 2020; 78: 331-334.

23. Birnkrant DJ, Bushby K, Bann CM, et al. Diagnosis and management of Duchenne muscular dystrophy, part 1: diagnosis, and neuromuscular, rehabilitation, endocrine, and gastrointestinal and nutritional management. Lancet Neurol 2018; 17: 251-267.

24. Birnkrant DJ, Bushby K, Bann CM, et al. Diagnosis and management of Duchenne muscular dystrophy, part 2: respiratory, cardiac, bone health, and orthopaedic management. Lancet Neurol 2018; 17: 347-361.

25. Birnkrant DJ, Bushby K, Bann CM, et al. Diagnosis and management of Duchenne muscular dystrophy, part 3: primary care, emergency management, psychosocial care, and transitions of care across the lifespan. Lancet Neurol 2018; 17: 445-455.

26. https://treat-nmd.org/family-care-guide/english-uk-dmd/ 